\title{
EL SUELO INDUSTRIAL COMO FACTOR DE OPORTUNIDAD Y RESILIENCIA EN ESPACIOS MINEROS Y RURALES DE LEÓN (ESPAÑA)
}

\author{
Paz Benito del Pozo \\ Universidad de León \\ paz.benito@unileon.es \\ ORCID iD: https://orcid.org/0000-0001-5492-3677 \\ Francisco José Diez Vizcaíno \\ Universidad de León \\ fjdv3@hotmail.com \\ ORCID iD: https://orcid.org/0000-0003-3740-4300
}

Recibido: 13/07/2018; Aceptado: 30/09/2019.

\begin{abstract}
Cómo citar este artículo/Citation: Benito del Pozo, P. y Diez Vizcaíno, F. J. (2019). El suelo industrial como factor de oportunidad y resiliencia en espacios mineros y rurales de León (España). Estudios Geográficos, 80 (287), e023. https://doi.org/10.3989/estgeogr.201940.020

RESUMEN: Se plantea analizar con un enfoque de geografía económica y método hipotético-deductivo el alcance de la estrategia pública de promoción de áreas empresariales en tres ámbitos comarcales de la provincia de León de dominancia rural y/o minera: Órbigo, Montaña y El Bierzo, considerando los desafíos de la crisis desencadenada en 2008 y la importancia del suelo industrial en la resiliencia de estos espacios. La hipótesis es que la oferta de suelo empresarial es un factor de resiliencia capaz de mejorar la capacidad de respuesta de espacios en declive y zonas rurales, ya que sería inductor de tres efectos positivos: reforzar la ventaja competitiva del territorio en coyunturas post-crisis, impulsar la innovación mediante proyectos empresariales de nuevo cuño, y mejorar las expectativas de progreso local. Los resultados obtenidos mediante técnicas cualitativas, uso de estadísticas y cartografía confirman la premisa de partida, pero también desvelan que los efectos positivos de los polígonos industriales y parques empresariales pueden ser de baja intensidad y producirse a ritmo desigual.
\end{abstract}

PALABRAS CLAVE: oferta de suelo industrial; declive espacios mineros; resiliencia en espacios rurales; desarrollo local.

\section{THE INDUSTRIAL LAND AS A RESILIENCE AND OPPORTUNITY ELEMENT IN MINING AND COUN- TRYSIDE AREAS IN LEÓN}

ABSTRACT: It is proposed to analyze with an economic geography and hypothetical-deductive method approach the scope of the public strategy of promotion of business areas in three regional areas of the province of León of rural and / or mining dominance: Órbigo, Montaña and El Bierzo, considering the challenges of the crisis unleashed in 2008 and the importance of industrial land in the resilience of these spaces. The hypothesis is that the development of industrial estates, that is, the offer of urbanized land for business activities, is a factor of resilience capable of generating opportunities in declining spaces and rural areas. The industrial land would be inductor of three positive effects: reinforce the competitive advantage of the territory in post-crisis situations, promote innovation through new business projects, and improve the expectations of local progress in terms of population and employment. The results obtained through the use of qualitative techniques, management of statistics and cartographic tools confirm the starting premise, but also reveal that the positive effects of industrial parks and business parks can be of low intensity and occur at an uneven pace.

KEY WORDS: supply of industrial land; decline of mining areas; resilience in rural areas; local development.

Copyright: @ 2019 CSIC. Este es un artículo de acceso abierto distribuido bajo los términos de la licencia de uso y distribución Creative Commons Reconocimiento 4.0 Internacional (CC BY 4.0). 


\section{INTRODUCCIÓN: LA PROMOCIÓN PÚBLICA DE SUELO INDUSTRIAL}

La creación de suelo industrial urbanizado en España responde, ante todo, al interés de generar una oferta de suelo para empresas que permita alcanzar los objetivos de la política industrial y de desarrollo económico a la escala que corresponda (local/urbana, regional, nacional), a la vez que se pretende conciliar dicho objetivo sectorial con los planteamientos cada vez más exigentes en materia de ordenación del territorio y planificación urbanística. En este proceso el protagonismo corresponde, en consecuencia, a los poderes públicos, más allá de que la iniciativa privada también desarrolle suelo industrial, animada con frecuencia por las ayudas procedentes de las Administraciones Públicas.

Un agente destacado desde fecha temprana es el propio Estado, que actúa a través de instituciones que irán tomando nombres diferentes desde los años de 1960 hasta llegar a la SEPES y la SEPI actuales. LoS Ayuntamientos, por su parte, ganan protagonismo en este tipo de actividad promotora en la etapa democrática, cuando asumen la responsabilidad de reservar terrenos para uso empresarial dentro del planeamiento urbanístico municipal; y por último, también sobresale la intervención de las agencias regionales, dependientes de los Gobiernos autonómicos, urgidos a participar en el mercado de suelo empresarial para paliar, entre otros, los efectos de la reconversión industrial de los años de 1980 y 1990 e impulsar las políticas de desarrollo regional.

Avanzado el primer decenio de 2000, a los objetivos antes señalados se suma, entre los agentes públicos, la preocupación por la innovación y el desarrollo de sistemas productivos locales, lo que va a propiciar el desarrollo de polígonos industriales y parques empresariales y tecnológicos destinados a captar y fijar inversión y actividades relacionadas con las nuevas tecnologías, la I+D+i y con los recursos endógenos (Méndez y Alonso 2002; Caravaca, 2002; Blasco y Martínez, 2002; Benito del Pozo, 2006; López Groh, 2009; Humbert, Molinero y Valenzuela, 2011). Una oferta de suelo industrial y tecnológico que antes de la crisis de 2008 podía considerarse insuficiente, pero que a partir de ese momento, por retracción de la demanda, se manifiesta como excedentaria (Albertos y Sánchez, 2014).

En la provincia de León se produce, sobre todo a partir de mediados de los años de 1990, una eclosión de polígonos industriales, repartidos por los diferentes ámbitos territoriales (comarcas mineras, zonas rurales y áreas urbanas) y con un balance que en el año 2006 arrojaba la cifra de 52 áreas empresariales, con una superficie en conjunto de 1.511 hectáreas. Por etapas, desde 1960 hasta 1989 se había construído el $21 \%$ de los polígonos leoneses; en la década de 1990 se creó el 44,5\% y entre 2000 y 2006 el 34, 5\% restante, con una tendencia al aumento de su tamaño medio, una mayor difusión en el territorio y mayor implicación de los agentes públicos, promotores del $85 \%$ del total de suelo industrial ofertado (Peiret, 1992; Benito del Pozo 2006; González y Benito 2008). Este crecimiento de la oferta de suelo industrial se explica por la combinación de varios factores: ayudas oficiales a zonas mineras y espacios desfavorecidos; una política de difusión de la actividad industrial más firme y coherente con los principios de una ordenación equilibrada del territorio; y unas mejores expectativas económicas para la provincia (Martínez y Luengo, 2004; Benito del Pozo, 2011; Pascual, 2012; Manero, 2012; González y Benito, 2013-2014; López, Escudero y Placer, 2017).

La crisis desencadenada en 2008 va a suponer, por su parte, un freno en general al desarrollo de nuevo suelo industrial y un estancamiento de la demanda, lo que se aprecia en el conjunto de los ámbitos comarcales leoneses y en espacios tanto urbanos como rurales y de montaña (González y Benito, 20132014; Benito del Pozo, 2017). En relación con este hecho, se pone de manifiesto un fenómeno de relevancia territorial: en ciertas zonas rurales y mineras de León, dotadas de una oferta de suelo industrial que apenas despertó interés entre la demanda antes de la crisis de referencia, dicho recurso se perfila como una ventaja territorial y como un factor de resistencia local cuando dicha crisis comienza a remitir a escala regional y nacional. Esto nos plantea algunos interrogantes: ¿Qué papel desempeña el suelo industrial en la superación de una coyuntura de crisis aguda en territorios que en sí mismos son periféricos o están lastrados por la atonía económica y la despoblación? ¿Son los polígonos industriales un factor de resiliencia en zonas mineras y rurales? ¿La oferta de suelo industrial garantiza la resiliencia en cualquier territorio? Las respuestas a estas cuestiones se buscan, como a continuación se expone, en el marco teórico y de discusión del desarrolo local y la resiliencia territorial.

Desde el punto de vista metodológico el análisis de la promoción pública de suelo industrial y su repercusión territorial requiere el uso de fuentes de diversa naturaleza, en particular la revisión de la 
bibliografía académica especializada, con especial mención de los trabajos geográficos, y la consulta de documentos e informes oficiales, la mayoría de ellos disponibles en la Red; una fuente secundaria interesante es la prensa económica y local, que permite obtener una visión del alcance que la sociedad otorga a este tipo de actuaciones, en las que se ven implicados agentes diversos (políticos, empresarios, sindicatos). No menos importante es el trabajo de campo, necesario para obtener información y datos sobre el terreno que completan las fuentes antes citadas.

En el plano teórico cobra especial protagonismo el concepto de resiliencia. En el contexto actual de fuerte competitividad entre los territorios, inestabilidad económica e incertidumbre social emerge el concepto de resiliencia territorial, un enfoque de éxito en la literatura reciente para referirse a la desigual capacidad de respuesta de los territorios frente a cambios drásticos o crisis agudas (Pike, Dawley y Tomaney, 2010) y para adaptarse a un nuevo contexto mediante estrategias orientadas a superar los procesos de declive y revertirlos (Méndez, 2012). De esta forma, un espacio resiliente no sería exitoso solo a corto plazo, sino que mantendría esta condición en el tiempo, haciendo frente a la inevitable adaptación que requieren los cambios en la economía global, lo que repercute en las políticas sectoriales y territorial.

Otro aspecto metodológico reseñable es el análisis Dafo, que se aplica por su valor sintético acerca de la situación de origen o de partida de los espacios rurales y mineros en crisis, y de las potencialidades que ofrecen a partir de recursos y acciones que entran en juego en la nueva dinámica territorial que introduce la oferta de suelo para empresas.

\section{LAS INFRAESTRUCTURAS INDUSTRIALES EN EL DESA- RROLLO LOCAL Y SU VÍNCULO CON LA RESILIENCIA}

Son numerosos los trabajos y publicaciones geográficas que se han ocupado del impacto de la reconversión en los espacios mineros (tipificados a partir de ese proceso como áreas periféricas) y las políticas que a lo largo del siglo XXI han tratado de diversificar la economía de esos terrerritorios con el fin de atajar una crisis aún sin resolver en su totalidad, lo que explica que este tema siga suscitando el interés de los investigadores y se abran líneas nuevas de interpretación que ponen el acento en la diversificación del tejido productivo, la sostenibilidad, la patrimonialización de la herencia minero-industrial y el turismo cultural, así como el desarrollo de una oferta cualificada de suelo industrial y tecnológico como recur- so para reactivar terrenos abandonados y crear oportunidades de negocio y empleo (vid. Revue Géographique de l'Est, 2017).

Igualmente sigue abierto el debate sobre los espacios rurales y el desarrollo local, y los aspectos asociados a este enfoque, como son la ordenación del territorio, el impacto de la innovación, las redes de transporte, el turismo o los programas comunitarios (Plaza Gutiérrez, 2005; Maya Frades, 2008; Moyano, 2009; Cortizo et al., 2014); preocupaciones que, por otra parte, ya centraban la discusión de los expertos desde tiempo atrás (Caravaca, 2002; Asociación de Geógrafos Españoles, 2004) y que ganaron profundidad al unirse al discurso de la sostenibilidad, planteado en toda su dimensión desde el año 2000 con un enfoque que sitúa en primer plano la globalización y pone el acento en las incertidumbres y las potencialidades que se vislumbran para los espacios rurales, lo que incluye cierta atención a las dotaciones de suelo urbanizado que fortalecen el tejido empresarial (García Pascual, 2001).

Es, sin embargo, en el contexto de los sistemas productivos locales donde se evidencia con más nitidez la importancia de los equipamientos industriales (suelo industrial y terciario para las pymes), pues una concentración de empresas competitivas en áreas rurales reclama un espacio urbanizado específico, con accesos y servicios que apoyen el desarrollo empresarial y los procesos de innovación en todos sus frentes: gestión, producto y proceso (Caravaca, 2002; Méndez y Alonso, 2002; Calderón y Pascual, 2009; Sánchez, Gallardo y Ceña, 2014).

En el caso de Castilla y León, los estudios auspiciados por el Gobierno autonómico sobre el futuro de la economía regional y las perspectivas de los espacios rurales y mineros ponen el acento, desde mediados de los años 90, en la necesidad de disponer de suelo industrial como mecanismo de respuesta a la crisis de las actividades tradicionales (minería del carbón, producción de energía, agricultura, ganadería). Se impone el discurso de que se trata de un factor de localización que puede favorecer procesos endógenos de industrialización, desarrollo de la agroindustria y fijación de actividades de nueva tecnología, ayudando a frenar la despoblación y a crear empleo (Junta de Castilla y León, 1998). En esta misma óptica institucional, un segundo estudio de diagnóstico, promovido por la Junta de Castilla y León al inicio del nuevo milenio, insiste en la idea de que "a pesar de las actuaciones inversoras realizadas cuya finalidad es propiciar el desarrollo, existe la percepción de que 
(...) las zonas eminentemente rurales, que en su mayoría coinciden con áreas montañosas (...), presentan un cierto grado de aislamiento y una serie de deficiencias o limitaciones en la prestación de servicios básicos y en la dotación de infraestructuras" (JCyL, 2002:17), carencias que apuntan expresamente a la oferta de suelo industrial.

Tales deficiencias tratarán de ser resueltas con un Plan Especial de Actuación en las Áreas Periféricas que incluye para el período 2002-2006 un eje prioritario de actuación centrado en el "Desarrollo Empresarial" y en concreto en "Promover la creación de infraestructuras que faciliten el desarrollo de los procesos productivos" (JCyL, 2002:40). Aquí cabe situar el origen del más firme compromiso oficial con la creación de polígonos y parques empresariales en León antes de la crisis de 2008, así como el reconocimiento explícito del papel favorable que desempeña el suelo industrial en las oportunidades de desarrollo rural y espacios mineros, donde además convergen las ayudas específicas al carbón y los proyectos de diversificación productiva (ibídem).

Asimismo, debe tomarse en consideración lo que algunos autores denominan el enfoque Leader, "una herramienta fundamental para tratar de dinamizar los territorios que presentan más debilidades, pero al mismo tiempo disponen de un conjunto de potencialidades para diversificar ingresos (...) y como base de nuevos yacimientos de empleo" (Sánchez e Hidalgo, 2017:186). Dicha iniciativa comunitaria afectó en el período 2000-2006 (Leader+ y Prodecal) a casi toda la provincia de León, con proyectos e inversiones específicas destinados al fomento y apoyo a las pymes y servicios, con el balance de 162 proyectos y una inversión media por proyecto superior a los 81.000 euros (Sánchez e Hidalgo, 2017). También estas acciones apuntalan la idea de la importancia de una oferta de suelo empresarial para acoger iniciativas de desarrollo rural, pues las pymes buscan este recurso en su entorno, que actúa como factor de localización (Martínez y Luengo, 2005).

Por lo que respecta al marco interpretativo de la resiliencia -tomado el concepto tanto en su acepción más amplia (Solana, 2016), como en la más acotada de resiliencia territorial, a saber: "la capacidad que poseen algunos territorios (ciudades, regiones, áreas rurales, etc.) para preparar, resistir o adaptarse a situaciones de shock (crisis económica, financiera, social o política; desastres naturales, ecológicos, industriales o epidemiológicos; cambio climático; cambio tecnológico; etc.) que inciden sobre los elementos del territorio, la población y sus actividades" (Sánchez, Gallardo y Ceña, 2014: 28)-, dicho enfoque parece adecuado para explicar cómo la oferta de suelo empresarial en áreas desfavorecidas, mineras y rurales en nuestro caso, cumple la función de mejorar la capacidad de respuesta de estos territorios, inmersos en procesos de crisis recurrentes (y con frecuencia solapadas). En efecto, según algunos estudios sobre resiliencia, entre los factores impulsores de procesos de cambio en las áreas rurales destacan, entre otros, las infraestructuras económicas (Sánchez, Gallardo y Ceña, 2014). Y la promoción de suelo industrial de calidad es, como se ha dicho, uno de los instrumentos de la política económica que busca desencadenar procesos de impulso territorial.

\section{RESULTADOS: PLANIFICACIÓN, DESARROLLO Y CAPACI- DAD DE IMPULSO TERRITORIAL DEL SUELO EMPRESARIAL EN ZONAS RURALES Y MINERAS DE LEÓN}

Castilla y León no dispone, a diferencia de otras CC AA, de un Plan de Suelo Industrial que defina los objetivos, desarrollos urbanísticos y financiación de este tipo de suelo de uso económico para períodos revisables de cuatro años, un instrumento vinculante para los Gobiernos regionales que de existir permitiría conocer con detalle la estrategia oficial y las acciones planificadas con relación a las infraestructuras de apoyo a la industria y las empresas en el ámbito territorial catellano y leonés. Esta ausencia o vacío en materia de suelo industrial se suple con lo dispuesto en las llamadas Directrices Esencialesde Ordenación del Territorio, aprobadas en 2008 (vid. Ley 3/2008, de 17 de junio, de aprobación de las Directrices Esenciales de Ordenación del Territorio de Castilla y León) y que proponen los siguientes objetivos:

1. Crear suelo industrial con flexibilidad de uso

2. Favorecer los medios innovadores

3. Responder con agilidad a la demanda de suelo industrial

4. Fortalecer las redes territoriales de apoyo empresarial

5. Especial atención a los espacios urbanos y núcleos rurales

Pese al interés de este instrumento, su contenido no suple lo que sería un verdadero plan regional de suelo industrial, donde se indicarían los desarrollos concretos de suelo y el volumen y las fuentes de inversión previstos en plazos temporales concretos (González y Benito, 2013-2014; Benito del Pozo, 2017). Esta 
situación refuerza el papel de los Ayuntamientos en la planificación del suelo para empresas, una realidad que puede condicionar ciertos objetivos de la política regional pues el equilibrio intraprovincial puede verse comprometido por falta de un criterio supramunicipal y/o de escala provincial/regional vinculante (Manero, 2012; Blasco y Martínez, 2002; Benito del Pozo, 2017).

De hecho, en las zonas rurales y de montaña leonesas, estas últimas de tradición minería, la creación de polígonos industriales ha dependido en buena medida de los intereses municipales, que bien de forma directa, o a través de entes regionales y/o al amparo de ayudas oficiales al sector del carbón, han generado la mayor oferta de suelo en la provincia, en sí misma un factor de desarrollo y oportunidad allí donde la disponibiliad de parcelas mantiene activo el mercado de suelo industrial (Benito del Pozo, 2017). Un reciente diagnóstico provincial (López Trigal et al., 2017) confirma la capacidad impulsora de esta dotación para empresas, que, sin embargo, es preciso analizar en el caso de cada polígono y contexto territorial para establecer su impacto (Tabla 1).
A tal fin se plantea el análisis de los polígonos industriales de los municipios de Villadangos del Páramo, Carrocera y Cubillos del Sil, seleccionados porque representan la estrategia oficial de industrialización inducida en León y por pertenecer a ámbitos territoriales contrastados, que brindan diversidad casuística y manifiestan rasgos geográficos que permiten extrapolar impactos y generalizar resultados (Tabla 2). La escala de anális de los parámetros básicos de la investigación (evolución de la población, tasa de desempleo) es comarcal/municipal, lo que unido al análisis de la consolidación de los polígonos permitirá apreciar la situación relativa de los municipios con polígono industrial frente al entorno sin este recurso.

Los polígonos analizados (Tabla 3) son asentamientos planificados desde su origen mismo, de promoción pública e incluidos en la modalidad de polígono industrial, ajustados a la definición que la literatura hace de esta tipología: espacios delimitados, con un perímetro que marca el uso de suelo industrial y urbanizados según un parcelario regulado por la urbanística municipal. Son, además, espacios favorecidos

TABLA 1

ANÁLISIS DAFO APLICADO AL SUELO INDUSTRIAL EN LEÓN

\begin{tabular}{|c|c|}
\hline DEBILIDADES & AMENAZAS \\
\hline $\begin{array}{l}\text { - Desequilibrio comarcal en la oferta de suelo industrial. } \\
\text { - Falta de interés por el lado de la demanda en suelo } \\
\text { industrial alejado de las grandes ciudades. } \\
\text { - Alto precio del suelo para las empresas de todo tipo. } \\
\text { - Comercialización poco efectiva/rentable del suelo } \\
\text { industrial y logístico. } \\
\text { - Escasa accesibilidad de los espacios logísticos. } \\
\text { - Dualismo territorial: procesos de crecimiento polarizados } \\
\text { en Ponferrada y León. }\end{array}$ & $\begin{array}{l}\text { - Desarrollo desigual del territorio provincial. } \\
\text { - Atrofia del tejido empresarial en zonas rurales y de montaña. } \\
\text { - Crecimiento desequilibrado de la oferta de suelo empresarial. } \\
\text { - Deterioro de la calidad de las infraestructuras industriales } \\
\text { (todo tipo de polígonos y parques). } \\
\text { - Atraso de los municipios sin suelo para empresas. } \\
\text { - Mala accesibilidad en espacios no urbanos. } \\
\text { - Pérdida de oportunidades en la logística del noroeste } \\
\text { peninsular. }\end{array}$ \\
\hline FORTALEZAS & OPORTUNIDADES \\
\hline $\begin{array}{l}\text { - Dinamismo de las áreas urbanas de Ponferrada y León } \\
\text { - Existencia de universidades y centros de investigación } \\
\text { ligados al tejido productivo. } \\
\text { - Apoyo oficial al desarrollo de áreas empresariales en } \\
\text { zonas de montaña y minería. } \\
\text { - Medidas locales de estímulo a las actividades alternativas } \\
\text { en espacios rurales } \\
\text { - Dotación de suelo empresarial en ámbitos de demanda } \\
\text { alta. } \\
\text { - Amplio peso de los Ayuntamientos en la promoción de } \\
\text { suelo empresarial. } \\
\text { - Compromiso de las autoridades locales con el desarrollo } \\
\text { de espacios productivos }\end{array}$ & $\begin{array}{l}\text { - Posicionar a León y Ponferrada como centros logísticos de } \\
\text { primer orden en el norte peninsular } \\
\text { - Potenciar la red de trasportes terrestres para lograr el } \\
\text { reequilibrio territorial. } \\
\text { - Lograr una red de polígonos y parques empresariales } \\
\text { densa y de calidad. } \\
\text { - Reforzar los vínculos entre espacios productivos y logística. } \\
\text { - Aumentar la competitividad de los espacios urbanos y } \\
\text { rurales con acciones de suelo empresarial y logístico } \\
\text { - Ampliar el área de influencia provincial a través de la } \\
\text { oferta de suelo industrial. } \\
\text { - Ampliar la importancia logística provincial para multiplicar } \\
\text { tráficos. }\end{array}$ \\
\hline
\end{tabular}

Fuente: Tomado de López Trigal, Escudedo y Placer, 2017: 289. 
TABLA 2

COMARCAS Y MUNICIPIOS ANALIZADOS: EXTENSIÓN Y POBLACIÓN

\begin{tabular}{|c|c|c|}
\hline MUNICIPIOS ÁMBITO ÓRBIGO & Superficie $\left(\mathrm{km}^{2}\right)$ & Población 2016 \\
\hline Villadangos del Páramo & 44,93 & 1.096 \\
\hline Santa Marina del Rey & 45,61 & 1.992 \\
\hline Benavides de Órbigo & 34,05 & 2.587 \\
\hline Turcia & 31,99 & 1.039 \\
\hline Carrizo de la Ribera & 41,85 & 2.335 \\
\hline MUNICIPIOS BIERZO RURAL & Superficie $\left(\mathrm{km}^{2}\right)$ & Población 2016 \\
\hline Cubillos del Sil & 53,41 & 1.813 \\
\hline Toreno & 103,53 & 3.304 \\
\hline Cabañas Raras & 19,11 & 1.344 \\
\hline MUNICIPIOS MONTAÑA-LUNA & Superficie $\left(\mathrm{km}^{2}\right)$ & Población 2016 \\
\hline Carrocera & 65,98 & 501 \\
\hline Rioseco de Tapia & 72,19 & 378 \\
\hline Santa María de Ordás & 45,58 & 338 \\
\hline Los Barrios de Luna & 94,29 & 315 \\
\hline Cuadros & 109,7 & 2.000 \\
\hline Soto y Amío & 69,19 & 830 \\
\hline
\end{tabular}

Fuente: Elaboración propia.

TABLA 3

DATOS BÁSICOS DE LOS POLÍGONOS INDUSTRIALES DE CARROCERA, VILLADANGOS Y CUBILLOS DEL SIL (LEÓN)

\begin{tabular}{|c|c|c|c|c|c|c|}
\hline Área empresarial & $\begin{array}{l}\text { Municipio/ } \\
\text { Comarca }\end{array}$ & Ente promotor & $\begin{array}{l}\text { Tipo de } \\
\text { promoción }\end{array}$ & $\begin{array}{l}\text { Año de } \\
\text { creación }\end{array}$ & $\begin{array}{l}\text { Superficie } \\
\left(\mathrm{m}^{2}\right)\end{array}$ & $\begin{array}{c}\text { Número } \\
\text { empresas } \\
\text { instaladas } \\
2016\end{array}$ \\
\hline $\begin{array}{l}\text { Polígono Industrial } \\
\text { "Los Avezales" }\end{array}$ & $\begin{array}{c}\text { Carrocera/ } \\
\text { Montaña-Luna }\end{array}$ & Ayuntamiento & Pública & 1999 & 143.000 & 5 \\
\hline $\begin{array}{l}\text { Polígono Industrial } \\
\text { "Villadangos" }\end{array}$ & $\begin{array}{c}\text { Villadangos del } \\
\text { Páramo/ } \\
\text { Órbigo }\end{array}$ & ADE (GESTURCAL) & Pública & 2002 & 2.000 .000 & 83 \\
\hline $\begin{array}{l}\text { Polígono Industrial } \\
\text { "El Bayo" }\end{array}$ & $\begin{array}{l}\text { Cubillos del Sil/ } \\
\text { El Bierzo }\end{array}$ & ADE (GESTURCAL) & Pública & 2002 & 1.354 .000 & 7 \\
\hline
\end{tabular}

Fuente: Elaboración propia.

por la dinámica de difusión por migración de la industria, lo que significa que en cierta medida participan del proceso general de relocalización a partir de las ciudades o cabeceras comarcales más próximas, además de propiciar la industrialización endógena (Benito del Pozo et al., 2014).

Con respecto a la estructura que ofrecen los agentes que impulsan la promoción de suelo empresarial en León, de la que son buena muestra los tres casos aquí analizados, destaca el hecho antes apuntado de que la mayor parte de los polígonos y parques em- presariales de la provincia son de iniciativa pública, con una intervención destacada de los Ayuntamientos, responsables del $67,3 \%$, de los polígonos y parques industriales, aunque también intervienen entidades estatales como SEPES Entidad Pública Empresarial de Suelo (un organismo adscrito al Ministerio de Fomento cuya misión es lograr el reequilibrio social y económico del territorio español a través de la promoción, adquisición y preparación de suelo para asentamientos industriales, terciarios y de servicios) y organismos dependientes del Gobierno regional, 
FIGURA 1

LOCALIZACIÓN Y DELIMITACIÓN DE LOS POLÍGONOS INDUSTRIALES EL BAYO, VILLADANGOS Y LOS AVEZALES
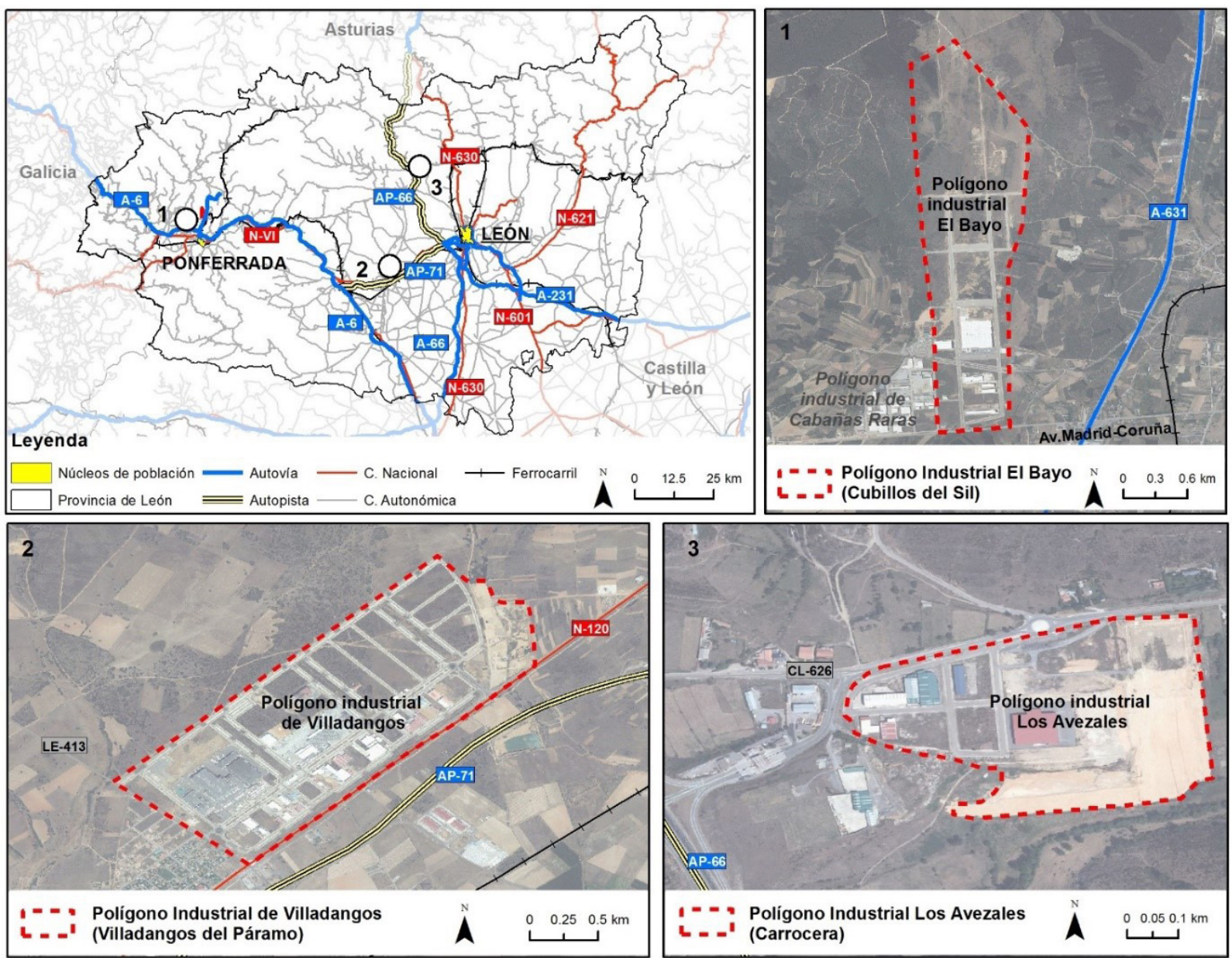

Fuente: Elaboración propia.

como es ADE Parques Tecnológicos y Empresariales, primer operador público de suelo de Castilla y León que nace de la fusión de dos empresas, Gesturcal y Parques Tecnológicos, dependientes de la Consejería de Economía y Empleo de la Junta de Castilla y León (http://www.aytovilladangosdelparamo.es/municipio/poligono/). Este hecho coincide con lo observado en otras Comunidades Autónomas y se explica, en primer lugar, por una razón simple: el suelo industrial no es un buen negocio (si se compara con el de uso residencial) y su riesgo es elevado, lo que ahuyenta al inversor privado. Por otra parte, la oferta de suelo para empresas se considera, como apuntamos más arriba, un instrumento de la política regional y local, de ahí que su promoción esté en manos de entes públicos (Benito del Pozo, 2006; González y Benito 2008; Benito del Pozo 2009).

\subsection{Villadangos: un polígono estratégico en un eje de comunicaciones clave}

El polígono industrial de Villadangos es un ejemplo de asentamiento empresarial planificado y ejecutado en función del interés del Gobierno regional de dotar al eje que conecta las ciudades de León y Astorga, de alto interés logístico, de un polo de atracción empresarial en terrenos de fácil urbanización y normativa urbanística favorable, amén de una óptima accesibilidad viaria y buena conexión aeroportuaria, una estrategia recurrente y en general eficaz, como demuestran otros estudios (Molina de la Torre, 2005). Está ubicado en las inmediaciones del núcleo rural del mismo nombre, en la comarca de Órbigo y se delimitó en el año 2002, un momento de notable actividad promotora por par- 
te del principal agente regional, GESTURCAL (Gestión Urbanística de Castilla y León, S.A), y a pesar de localizarse en un espacio de baja densidad de población y débil urbanización su emplazamiento, en el área de influercia urbana de León capital y sobre un eje clave en las comunicaciones transversales de la provincia, le otorga un alto interés logístico, como se aprecia en la Figura 2 y se deduce de los siguientes datos: (a) localización en el punto intermedio entre el eje León-Astorga (N-120) y la carretera local-comarcal de Villanueva de Carrizo (LE-413); (b) cercanía a la infraestructura ferroviaria que recorre transversalmente la provincia, concretamente a 500 metros del muelle de carga-descarga de la línea de ferrocarril Palencia-La Coruña; (c) apoyo directo sobre la autopista AP-71 León-Astorga que discurre paralela al polígono industrial y al eje de la $\mathrm{N}-120$. Se trata de una autopista de peaje con una longitud de $37 \mathrm{~km}$ y va desde la salida 143 de la A-66 hasta la salida 323 de la A-6. Tiene seis enlaces, todos ellos totales; y (d) está situado a seis kilómetros del aeropuerto provincial Virgen del Camino de León, lo que facilita las relaciones empresariales, la logística de los negocios y de los intercambios de bienes y servicios.

El interés para las actividades logísticas que reviste Villadangos explica su expansión y grado de ocupación empresarial, hasta llegar a convertirse en un asentamiento industrial de gran tamaño, 200 hectáreas de superficie total parcelada, el mayor de la provincia, y que oferta parcelas de diferente tamaño, pensadas para pymes con necesidades de suelo muy diferentes. Su mayor ventaja territorial son las conexiones viarias y la accesibilidad al entorno rural/periurbano al que sirve y a las ciudades de León (a $12 \mathrm{~km}$ ) y Astorga (a una distancia de 31 $\mathrm{km}$ ). El estudio de los servicios y dotaciones que ofrece permite constatar que el polígono incluye desde los suministros básicos (agua, red de alcantarillado, energía eléctrica, red de telefonía, gas natural) hasta la fibra óptica, un nivel de dotación que eleva la calidad media de los polígonos del entorno, un rasgo diferencial de la oferta que lo sitúa con ventaja en el mercado leonés del suelo industrial (González y Benito, 2013-2014).

Desde su creación el polígono ha conocido tres fases de desarrollo urbanístico, pasando de una superficie de 98 hectáreas en la primera fase, consolidada en 2006, a un total de 200 ha en la actualidad, resultado de la ejecución desde 2008 de las fases segunda y tercera, una expansión que se interpreta como capacidad territorial para la actividad productiva y factor de resileiencia. Según se desarrolla su parcelario, el proceso de comercialización se afianza y esto eleva el grado de ocupación del asentamiento, que en 2015 concentraba 83 empresas, tanto nacionales (fabricación de muebles, transportes, distribución, metalurgia, motor..), como internacionales (sobresale la compañía danesa Vestas, que fabrica componentes de molinos eólicos). Asimismo cabe destacar la presencia desde el año 2010 del Centro Logístico de Mercadona, una empresa líder en el sector de la distribución que realiza sus operaciones para el noroeste peninsular desde Villadangos y que se sirve de importantes inter-

FIGURA 2

PLANO PARCELARIO Y ESQUEMA DE CONEXIÓN DEL POLÍGONO DE VILLADANGOS CON SU ENTORNO

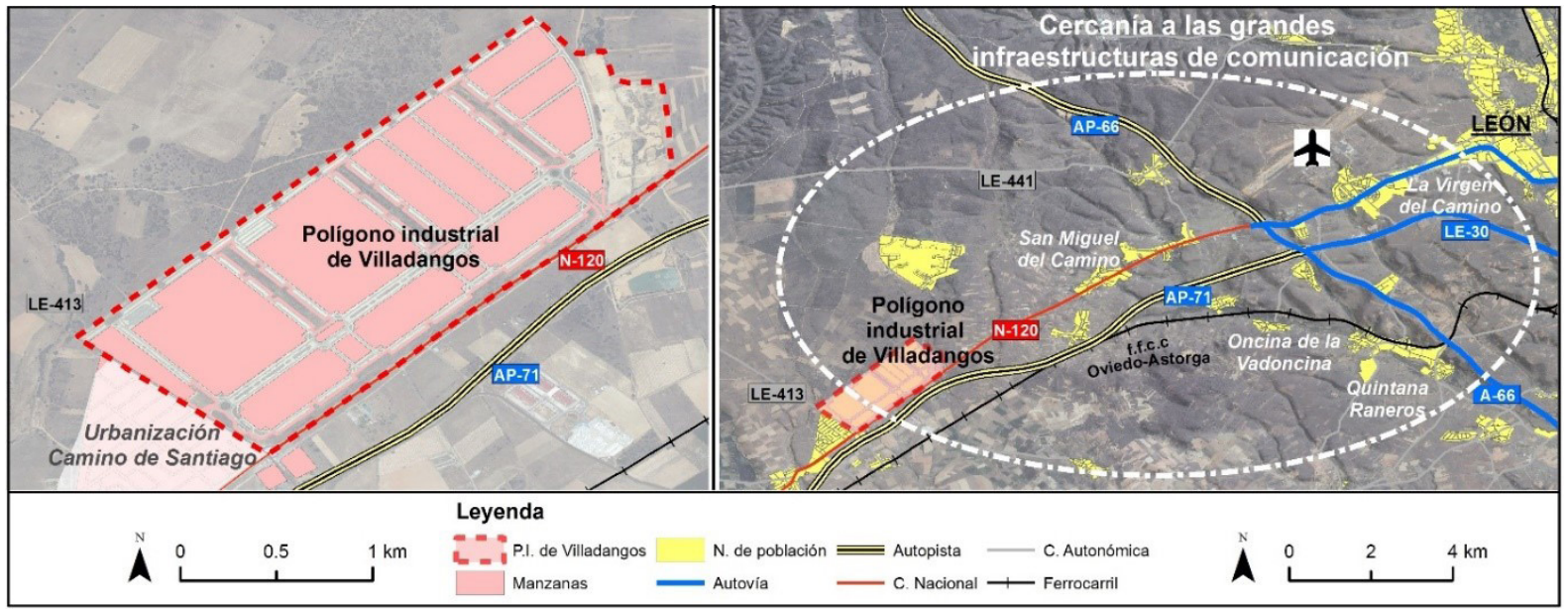

Fuente: Elaboración propia a partir de Base Topográfica Nacional a escala 1:100.000 y ortofotos del PNOA. 
proveedores: Lactiber (lácteos), Seprolesa (conservas vegetales), Font Agudes (agua mineral) y Cantos Blancos (huevos) (Rodríguez Lago, 2012). La innovación (y también el empleo) se verá reforzada a partir de 2019 con motivo de la puesta en marcha del Centro de Proceso de Datos (CPD) que la propia Mercadona está construyendo en una parcela de 7.359 metros cuadrados del polígono, con una inversión que se eleva a 4,3 millones de euros (vid. http://www.diariodeleon.es/ noticias/leon/mercadona-invierte-4-3-millones-cpdleon-preve-abrir-2019_1242761.html).

Desarrollar un área empresarial en este ámbito geográfico supuso asumir por parte del promotor una incertidumbre y un riesgo elevados, debido a indicadores y procesos desfavorables en la zona: despoblación, envejecimiento, altas tasas de desempleo, atonía productiva y tejido empresarial débil (JCyL, 2002). Antes de la puesta en marcha del polígono los municipios de la comarca experimentaban en conjunto y año tras año una disminución de población que rondaba el $2 \%$ de pérdida media anual y el $11 \%$ de pérdida media total desde 1998 (Figura 3). De igual forma, la evolución del porcentaje de población desempleada ofrecía valores poco halagüeños, según se deduce de los datos del INE (Figura 4).

La creación del polígono industrial va a propiciar un cambio de tendencia en el comportamiento de estos indicadores. En la Figura 3 se observa que desde el año 2003 la evolución demográfica de Villa- dangos con respecto a su entorno toma un rumbo opuesto, ofreciendo una dinámica de crecimiento positiva, aunque con altibajos (caídas puntuales en 2007, 2011-12 y 2014) que evidencian la crisis de fondo, pero que no impiden afirmar la tendencia al crecimiento y una mejor posición respecto del resto de municipios del Órbigo.

Algo similar sucede con la dinámica de cambio anual del paro, Figura 4, que presenta una tendencia de progreso ascendente desde comienzos de la década de 2000. Con el polígono industrial de Villadangos, si bien no se consigue invertir a corto plazo el ligero, aunque constante, incremento del porcentaje de población desempleada en la zona, se logra estabilizar el impacto de la crisis económica, lo que también puede interpretarse como evidencia de resiliencia, pues en el supuesto de no haber polígono industrial el desempleo hubiera sido mayor en volumen y ritmo. En el perío 2008-2012 el comportamiento de los indicadores de empleo son sin duda más favorables en Villadangos que en el resto del Órbigo.

En suma, a pesar de la coyuntura nacional e internacional recesiva el polígono industrial de Villadangos ha conseguido consolidarse como elemento regulador de estas pérdidas, favoreciendo que el municipio de Villadangos sufra de forma menos severa que el resto de sus vecinos los efectos de la crisis, al tiempo que sus oportunidades en la etapa de cambio de tendencia se ven incrementadas por este recurso territorial.

FIGURA 3

DINÁMICA DEMOGRÁFICA INTERCENSAL EN EL ÓRBIGO, 1998-2015

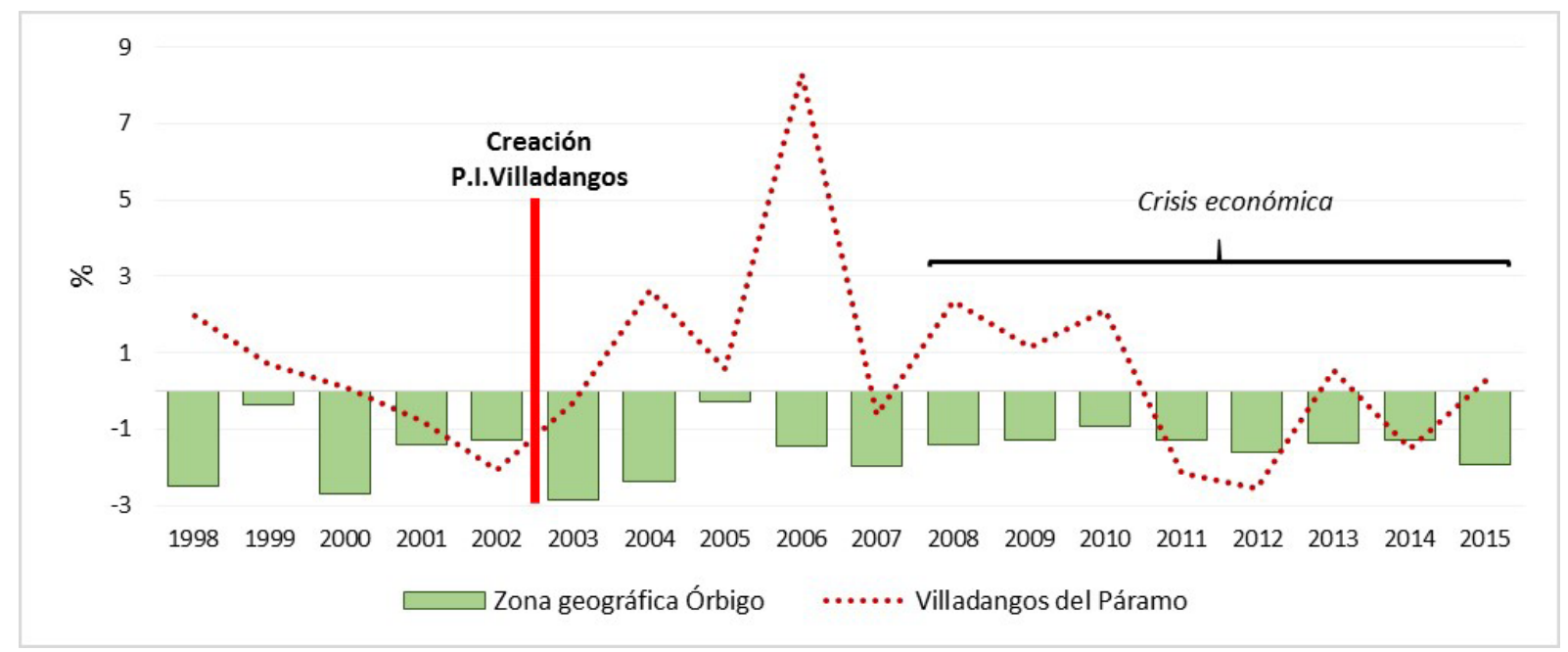

Fuente: Elaboración propia con datos del INE. 
FIGURA 4

EVOLUCIÓN DE LA TASA DE PARO REGISTRADO EN EL ÓRBIGO, 1997-2012

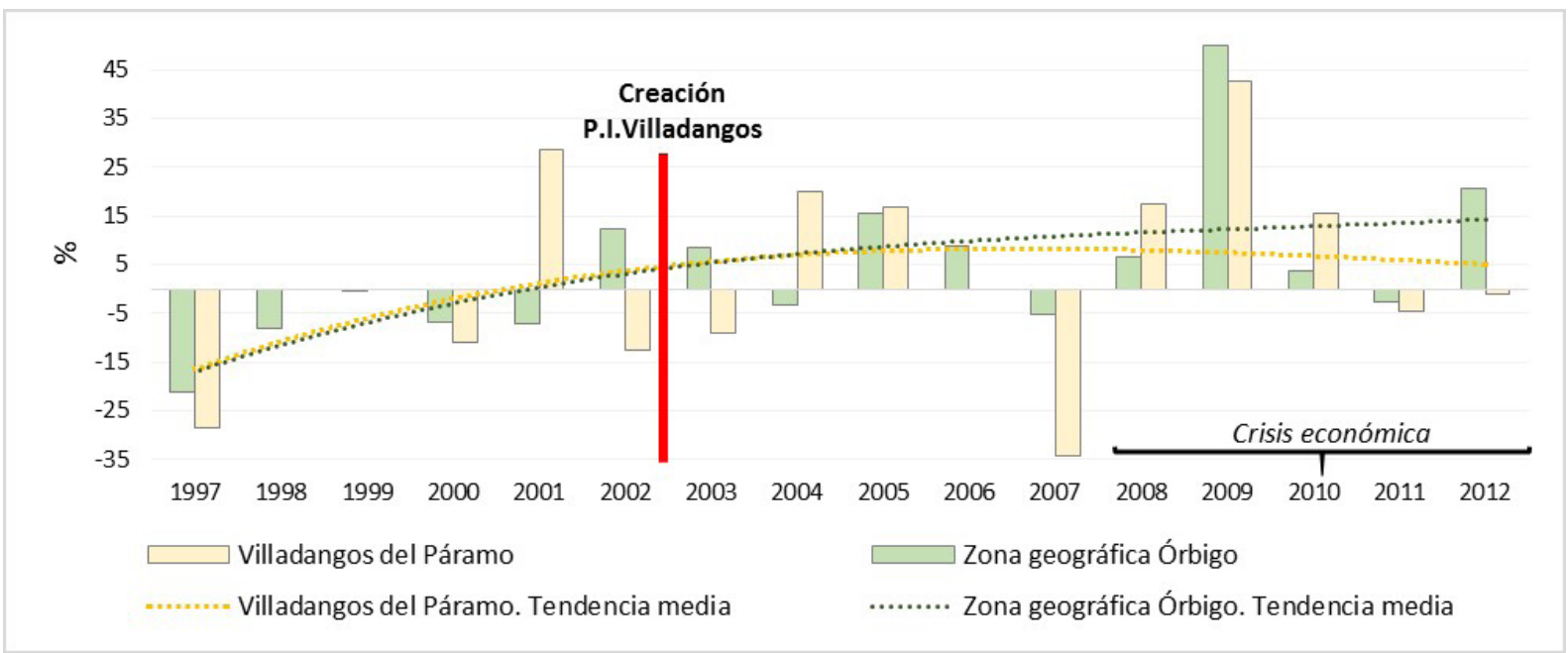

Fuente: Elaboración propia con datos del Anuario Económico de España "la Caixa".

\subsection{Cubillos del Sil: suelo industrial para la dinami- zación del Bierzo}

El área empresarial ubicada en el término municipal de Cubillos del Sil, el polígono de El Bayo, es otro de los ejemplos de asentamiento para actividades económicas con potencial dinamizador e innovador, en este caso situado en El Bierzo y en el área urbana de Ponferrada, ciudad de la que dista tan solo 10 $\mathrm{km}$. El Bierzo, un espacio de tradición empresarial y especializado en minería del carbón como recurso/ actividad principal hasta los años de 1990, ha sufrido en las últimas décadas un proceso de reconversión minera, con fuertes ajustes a la baja en el sector, que se ha tratado de compensar mediante políticas de diversificación económica, lo que ha supuesto la incorporación al tejido productivo de nuevas empresas del sector metalúrgico y de la construcción (subsector de la pizarra), así como otras vinculadas a la energía eólica y a la agroalimentación (Rodríguez y Cortizo, 2006). El proceso de impulso industrial se concentra sobre todo en Ponferrada, que actúa como centro difusor de sinergias de crecimiento que demandan, entre otras infraestructuras, suelo industrial urbanizado. Es en esta fase de acciones oficiales de dinamización industrial cuando se trata de impulsar un nuevo polígono con recursos procedentes de los Fondos Mineros (UE) y apoyo de la Junta de Castilla y León, localizado en el término municipal de Cubillos del Sil (vid. https://bit.ly/2OrSa2I). La proximidad a Ponferrada, ciudad de referencia en la comarca y considerada a escala regional un enclave logístico de primer orden, y la muena accesibilidad de Cubillos a las principales infraestructuras de transporte (en particular la autovía A-6), son los factores decisivos para desarrollar el polígono industrial El Bayo (González y Benito, 2013-2014). (Figura 5).

La delimitación de El Bayo se aprobó en 2002 y se comenzó a construir en el año 2005 con una superficie total prevista de 225 hectáreas y la previsión de urbanizar el conjunto del polígono en tres fases. La primera de ellas se inauguró en febrero de 2007 con unas 90 hectáreas; la segunda fase se construyó en 2012; pero la tercera y última fase está sin urbanizar (por lo que la superficie actual es de 135,4 hectáreas). El polígono tiene muy buenas comunicaciones con el entorno inmediato y remoto: la carretera CL-631 a un kilómetro y en dirección a Ponferrada enlaza a cuatro kilómetros con la autovía A6 (Figura 5). Desde el punto de vista de las dotaciones cuenta con red de agua potable, red de alcantarillado, infraestructura subterránea para el suministro de energía eléctrica, red telefónica y canalización subterránea de gas natural. Se trata de una zona favorecida por las subvenciones aplicables a los proyectos empresariales que promueven la Junta de Castilla y León y el Gobierno de España (Sánchez e Hidalgo, 2017) y, en particular, por las ayudas MINER (Rodríguez y Cortizo, 2006). Según los responsables municipales "uno de los logros que se consiguió cuando se construyó El Bayo fue que Cubillos se considerase municipio Miner en el ámbito 
FIGURA 5

EMPLAZAMIENTO Y PARCELARIO DEL POLIGONO EL BAYO (IZD.) Y ESTRATEGIA DE PLANIFICACIÓN (DCHA.)

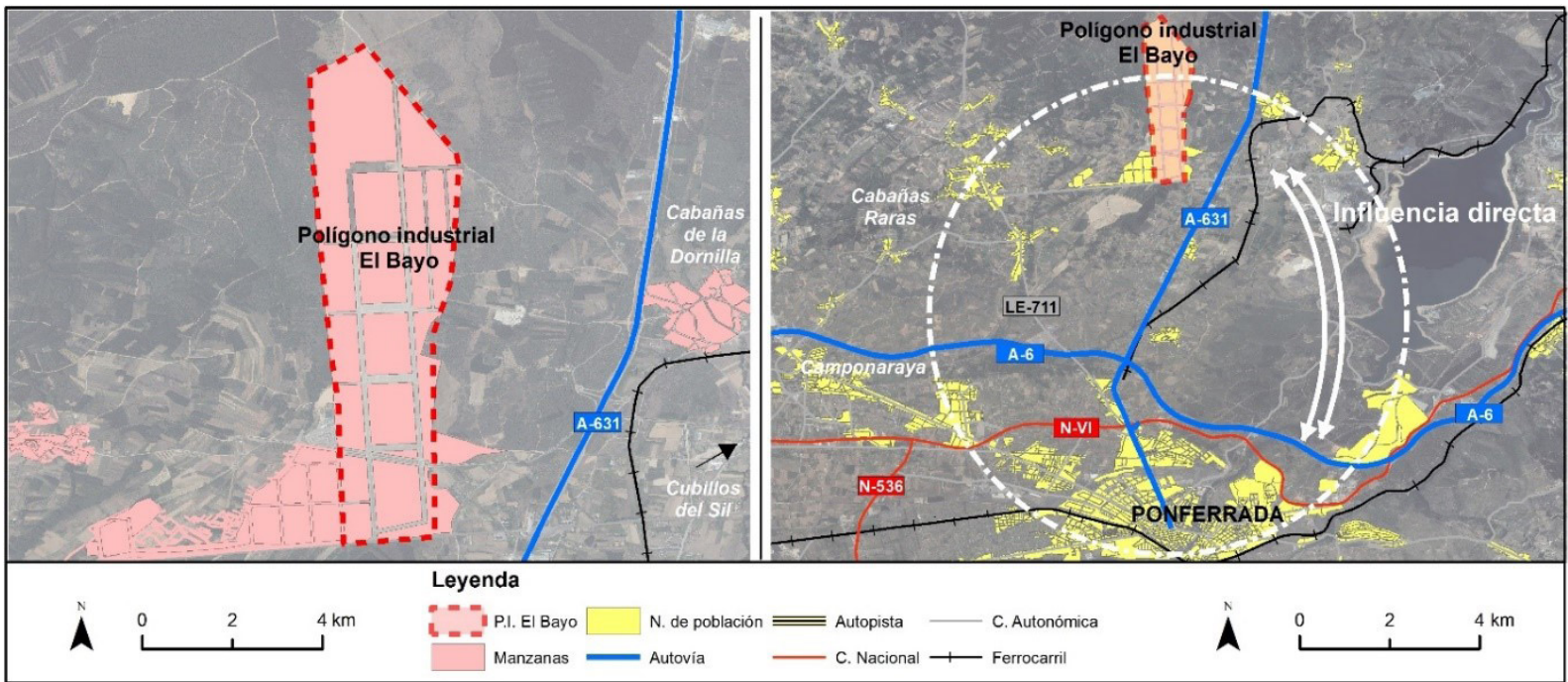

Fuente: Elaboración propia con datos de Base Topográfica Nacional a escala 1:100.000 y de las ortofotos del PNOA.

de actuación del polígono. Por lo tanto, las posibles industrias que se instalen o que se vayan a instalar en el futuro tendrán acceso a dichos Fondos" (vid. El Bierzo Digital, 26 de abril de 2012 https://www.elbierzodigital.com/el-bayo-sigue-aspirando-a-ser-el-motor-deel-bierzo-2/706).

Este polígono industrial, promovido también por GESTURCAL, no se limita a captar y fijar actividades en su entorno, sino que se proyecta como un imán para atraer inversión y empresas del noroeste peninsular, habida cuenta de su posición logística (Rodríguez Lago 2012). En 2007 el polígono era valorado por el propio alcalde como el principal motor económico local: "el polígono supone para un municipio relativamente pequeño como es Cubillos «más empleo, más actividad, más movimiento, más trabajo, más vida en el pueblo, más actividad en los restaurantes, más actividad en los alquileres de las viviendas (...)» (vid. Diario de León, 19/10/2007 http://www.diariodeleon. es/noticias/bierzo/gesturcal-adjudica-ampliacioncasi-900-000-metros-bayo_348396.html). Sin embargo, pese a las expectativas suscitadas, este polígono "minero" ofrece un bajo grado de consolidación. Su desarrollo urbanístico y comercialización fue lento e incompleto, y su nivel de ocupación es muy bajo (González y Benito, 2013-2014; Benito, 2017). Pese a que el polígono arrancó con la industria metalúrgica Ecalnor $\mathrm{XXI}$, que realizó una inversión de 4,2 millones de euros y creó 40 empleos directos, a la que siguieron otras inversiones, hasta sumar en 2012 un total de nueve empresas instaladas, el balance en 2016, es de siete empresas asentadas (Tabla 1).

El limitado éxito en la venta de este suelo industrial y el bajo nivel de consolidación empresarial se agudizó en los años de la crisis y solo tras tocar ésta fondo y apreciarse una cierta reactivación económica se han adoptado decisiones y compromisos de inversión relevantes para el polígono, el municipio y la comarca. En concreto, se está fraguando la instalación del Centro Cárnico de Alta Tecnología-Cicat, con una inversión de 50 millones de euros, la creación de unos 300 empleos directos y con una repercusión económica superior a 25 millones de euros anuales. El proyecto se anunció a finales de 2014, pero estuvo estancado hasta 2016, cuando se confirma la entrada de un socio oriental dispuesto a aportar financiación (vid. https:// www.lanuevacronica.com/la-carnica-icg-consiguefinanciacion-oriental-para-instalarse-en-cubillos).

Al igual que en el Órbigo, en el término municipal de Cubillos del Sil, previo a la creación del polígono industrial El Bayo, los marcadores socioeconómicos y demográficos eran negativos, con despoblación y aumento del paro. Entre los años 1998 y 2004 la pérdida de efectivos demográficos en este municipio fue del 6 $\%$, siendo aún más abultada y preocupante la evolución del porcentaje de población desempleada, que desde 1997 hasta 2004 manifiesta una caída media del $6 \%$ y total del $43 \%$. También en este caso el polígono industrial, ya en marcha en ese año, va a actuar como elemento dinamizador y factor de crecimiento 
local, al menos en los años previos a la crisis de 2008. Desde el punto de vista demográfico el crecimiento intercensal de la población en la zona de Cubillos del Sil experimenta, según se aprecia en la Figura 6, un fuerte incremento desde 2005 hasta el registro de 2015 (salvando los inevitables efectos negativos de la crisis internacional), alcanzando un $24 \%$ de incremento (porcentaje radicalmente opuesto al $3 \%$ de pérdida media que experimentan otros municipios del entorno rural del Bierzo en ese mismo periodo).

Con respecto a la evolución de la tasa de desempleo, reflejada en la Figura 7, si bien este indicador muestra un cambio en la tendencia de crecimiento a comienzos de la década de los 2000 (cuando el paro comienza a incrementarse de forma paulatina y constante), la puesta en marcha de El Bayo permite mantener algo más baja y tenue esta tendencia al alza del paro durante los años previos a la crisis económica, la cual altera la lógica evolutiva y el comportamiento del porcentaje de desempleados en la zona, ahora más alto en el término municipal de Cubillos del Sil, donde el tejido productivo generado gracias a los fondos mineros se resiente de la crisis y el polígono industrial deja de mostrarse, en ese momento, como un factor de crecimiento local.

Estos datos son expresivos del papel que desempeña la oferta de suelo industrial en espacios de limitado progreso, afectados en este caso por la reconversión minera. No se trata de un factor independiente del entorno, ni de un recurso por encima de coyunturas adversas; de hecho, en el período 2008-2012, con la crisis en auge, la oferta de suelo no es suficiente para frenar el paro ya que la inversión se estanca y no llegan nuevos proyectos empresariales. El polígono se paraliza y no será hasta que la crisis toque fondo y se esboce la recuperación, a partir de 2015, cuando se generen nuevas corrientes de oportunidad que se concretan en anuncios oficiales de nuevos proyectos, un hecho que indica un cambio de ambiente inversor.

\subsection{Carrocera: un polígono industrial municipal liga- do a la vía estructurante de alta capacidad AP-66}

El polígono industrial Los Avezales es otro ejemplo de asentamiento empresarial planificado que surge al calor de las ayudas a los espacios mineros y como parte de la estrategia oficial de dinamización económica y diversificación productiva que se ha comentado más arriba. El término municipal de Carrocera,al norte de la provincia de León, forma parte de un territorio también de tradición carbonera y con una población de 501 habitantes en 2016, en el ámbito comarcal que se denomina Montaña-Luna. Si bien su carácter de espacio de montaña y sus rasgos de área desfavorecida (JCyL, 2002) no parecen factores ventajosos a priori para vender parcelas de suelo industrial, concurre aquí un elemento espacial que refuerza las oportunidades de este municipio y le confiere una ventaja destacable: su posición respecto a la vía de alta capacidad AP-66 que vertebra de norte a sur la provincia y la conecta, hacia el norte, con Asturias y sus puertos de mar; y hacia el sur, con Madrid y el centro del país (Figura 8).

FIGURA 6

DINÁMICA DEMOGRÁFICA INTERCENSAL EN LA ZONA RURAL DEL BIERZO, 1998-2015

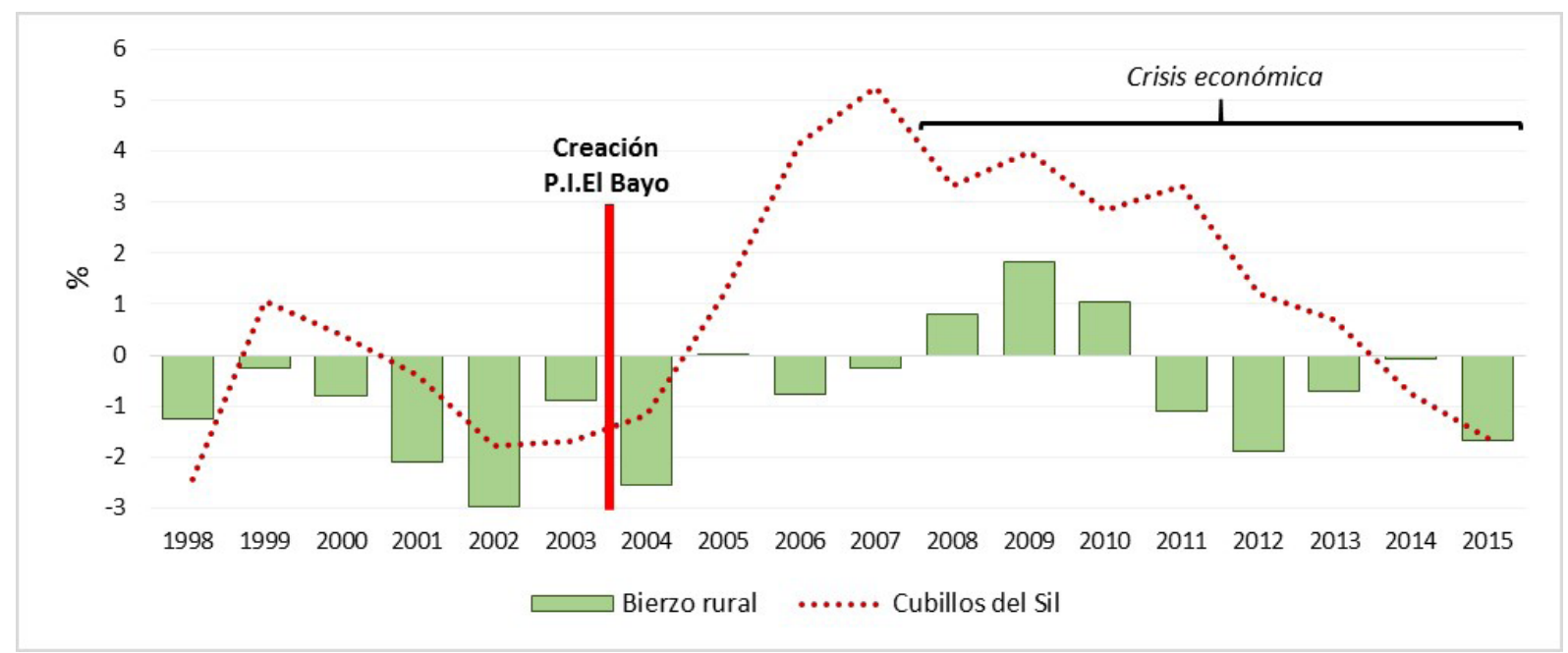

Fuente: Elaboración propia con datos del INE. 
EVOLUCIÓN DE LA TASA DE PARO REGISTRADO EN LA ZONA RURAL DE EL BIERZO, 1997-2012

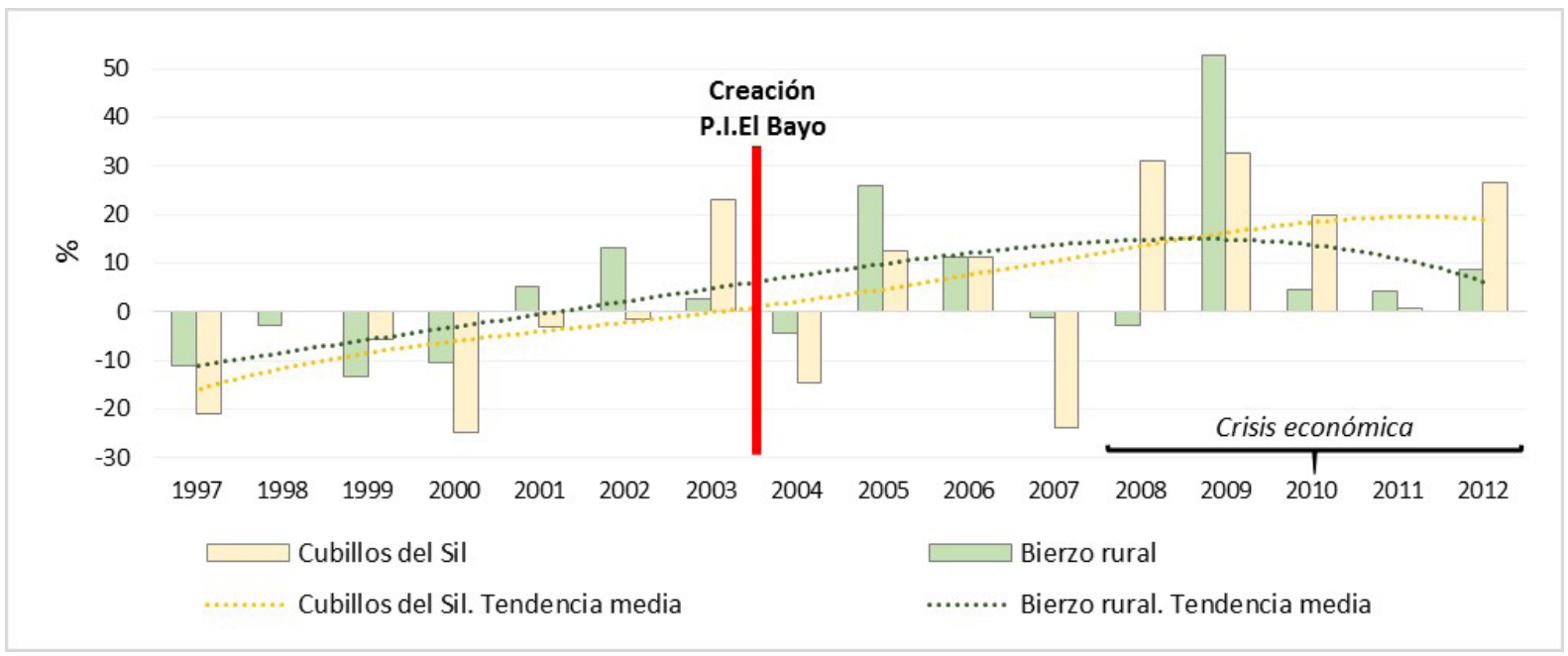

Fuente: Elaboración propia con datos del Anuario Económico de España “la Caixa”.

FIGURA 8

PLANO PARCELARIO BÁSICO (IZD.) Y LOCALIZACIÓN DEL POLÍGONO INDUSTRIAL LOS AVEZALES (DCHA.)

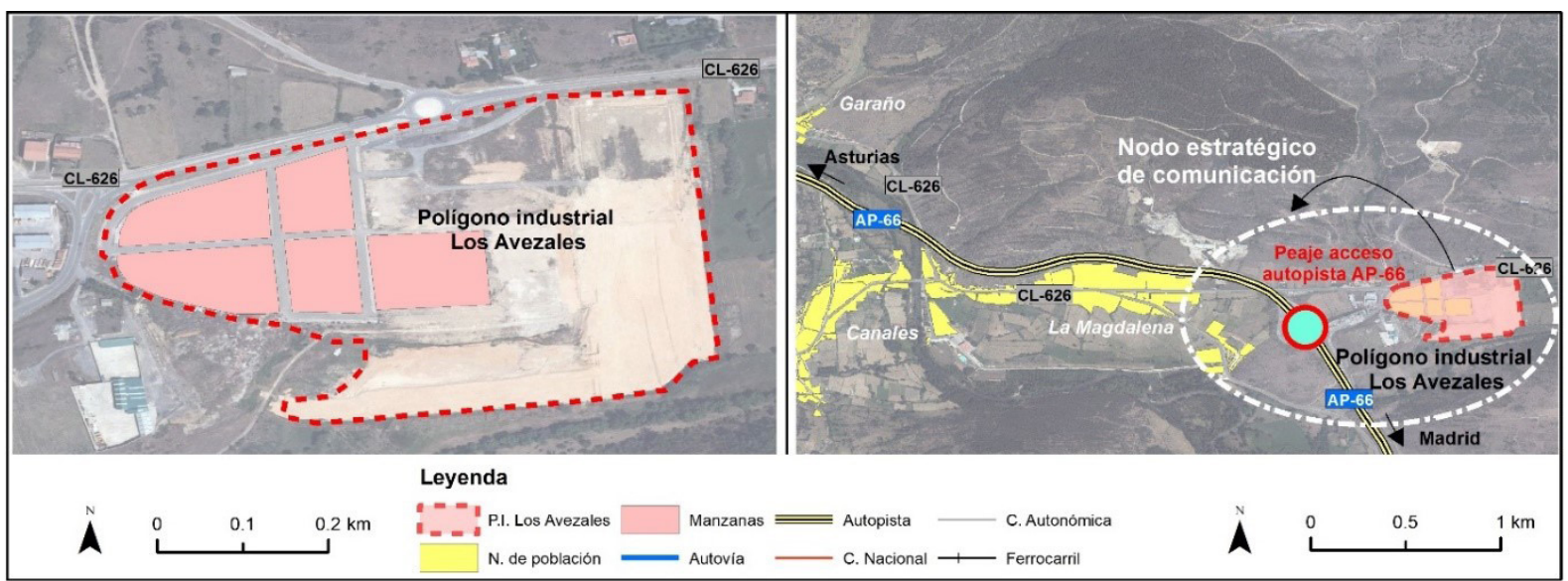

Fuente: Elaboración propia con datos de Base Topográfica Nacional a escala 1:100.000 y ortofotos del PNOA.

La posición estratégica de Carrocera con relación a la autopista AP-66 es, como se ha indicado, el factor clave para desarrollar un polígono industrial, concretamente entre los núcleos rurales de La Magdalena y Otero de las Dueñas, donde se halla uno de los puntos de peaje de la autopista de referencia, y a pocos kilómetros del nudo ferroviario de La Robla. En este caso es el Ayuntamiento el promotor del polígono de Los Avezales, que se delimita y desarrolla en 1999 con ayuda de Fondos Miner y una superficie total de 14,3 hectáreas, una dimensión que permite catalogar el asentamiento de "pequeña zona empresarial", aunque proporcionada a las posibilidades y capacidad productiva de la zona.
Si bien la construcción de este polígono industrial tuvo un impacto socioeconómico y territorial positivo (González y Benito, 2013-2014), el proceso de consolidación ha sido lento y los resultados modestos, pues la nómina de empresas instaladas es de cinco en la actualidad (Tabla 1), relacionadas con recursos endógenos: destaca el tratamiento de hierbas aromáticas y la comercialización de tisanas, además de algunos negocios ligados a la alimentación elaborada y catering. El impacto territorial del polígono es limitado, entre otras razones, porque se trata de un asentamiento industrial muy dependiente del movimiento pendular de la población ocupada (la gente que trabaja en Los Avezales no reside, en su mayoría, en el municipio de 
Carrocera, procede del exterior, pero encuentran ventajas para desplazarse por carretera debido al óptimo acceso a la AP-66). Sin embargo, aunque su capacidad para fijar nueva población en la zona es débil, sí es una infraestructura económica que puede estabilizar población; o lo que es lo mismo, es un factor capaz de frenar la despoblación.

En la Figura 9 puede apreciarse el ritmo de evolución del crecimiento intercensal de la población en el término de Carrocera, cómo la dinámica demográfica de este espacio mantiene una constante evolutiva anual algo más viva y cambiante que la que se desprende de los datos de los municipios limítrofes, cuyo crecimiento mantiene una tendencia negativa hasta el momento actual, solo alterada en el año 2011, cuando es apreciable un ligero crecimiento intercensal de la población.

Para el análisis del desempleo en este ámbito de Montaña-Luna solo se dispone de datos fiables desde 2007, y ello tomando una fuente de información diferente de los casos anteriores, pues por razones de difícil comprensión el Anuario Económico "la Caixa” no aporta cifras sobre los municipios de esta zona. En consecuencia, la evolución del desempleo en Carrocera desde 2007 en adelante procede de ExpansiónDatosmacro. En la Figura 10 se observa, al inicio de la crisis, un retroceso suave del paro en Carrocera frente a un descenso algo más brusco en el comportamiento medio del resto de municipios del espacio comarcal. Sin embargo, el descenso en el municipio de Carrocera sigue una tendencia descendente continua frente a un estancamiento en valores algo más elevados en el resto de la zona, siendo más favorable al dinamismo territorial el comportamiento identificado donde se encuentra el polígono industrial.

En definitiva, pese a su modesta dimensión en superficie y alcance económico, el polígono industrial de Los Avezales demuestra capacidad de atracción y fijación de actividades que diversifican la producción y que se hubieran perdido o localizado fuera del municipio de no existir esta la oferta de suelo, con el consecuente agravamiento de la recesión acumulada desde la crisis del carbón y el impacto de la crisis financiara, que afectó, entre otros, a los transportes canalizados por la AP-66, de los que se beneficia Carrocera.

\section{CONCLUSIONES}

En los espacios mineros y rurales inmersos en una dinámica de atonía productiva y demográfica provocada por crisis sectoriales sucesivas y agravadas desde 2008, la oferta de suelo industrial urbanizado es un factor de oportunidad y de resiliencia, pues incrementa la capacidad del territorio para mejorar sus condiciones de desarrollo y progreso socioeconómico, a la vez que actúa como imán de la innovación, al propiciar la localización de actividades y empresas con algún componente innovador. La acción de los responsables públicos de generar una oferta de suelo industrial puede interpretarse como una respuesta de resiliencia territorial, en los términos que ha sido incorporado este enfoque al caso de León.

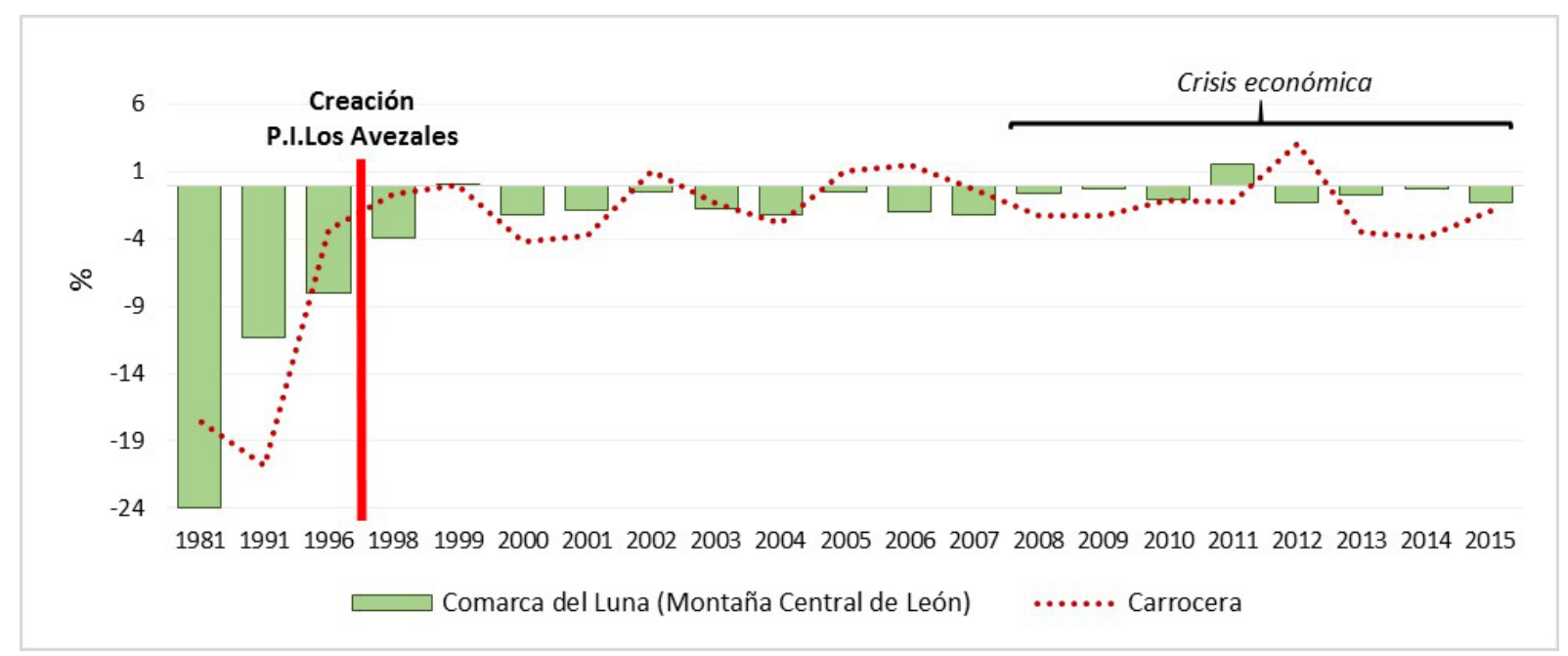

Fuente: Elaboración propia con datos del INE. 
FIGURA 10

EVOLUCIÓN DE LA TASA DE PARO REGISTRADO EN LA ZONA RURAL MONTAÑA-LUNA, 2007-2015

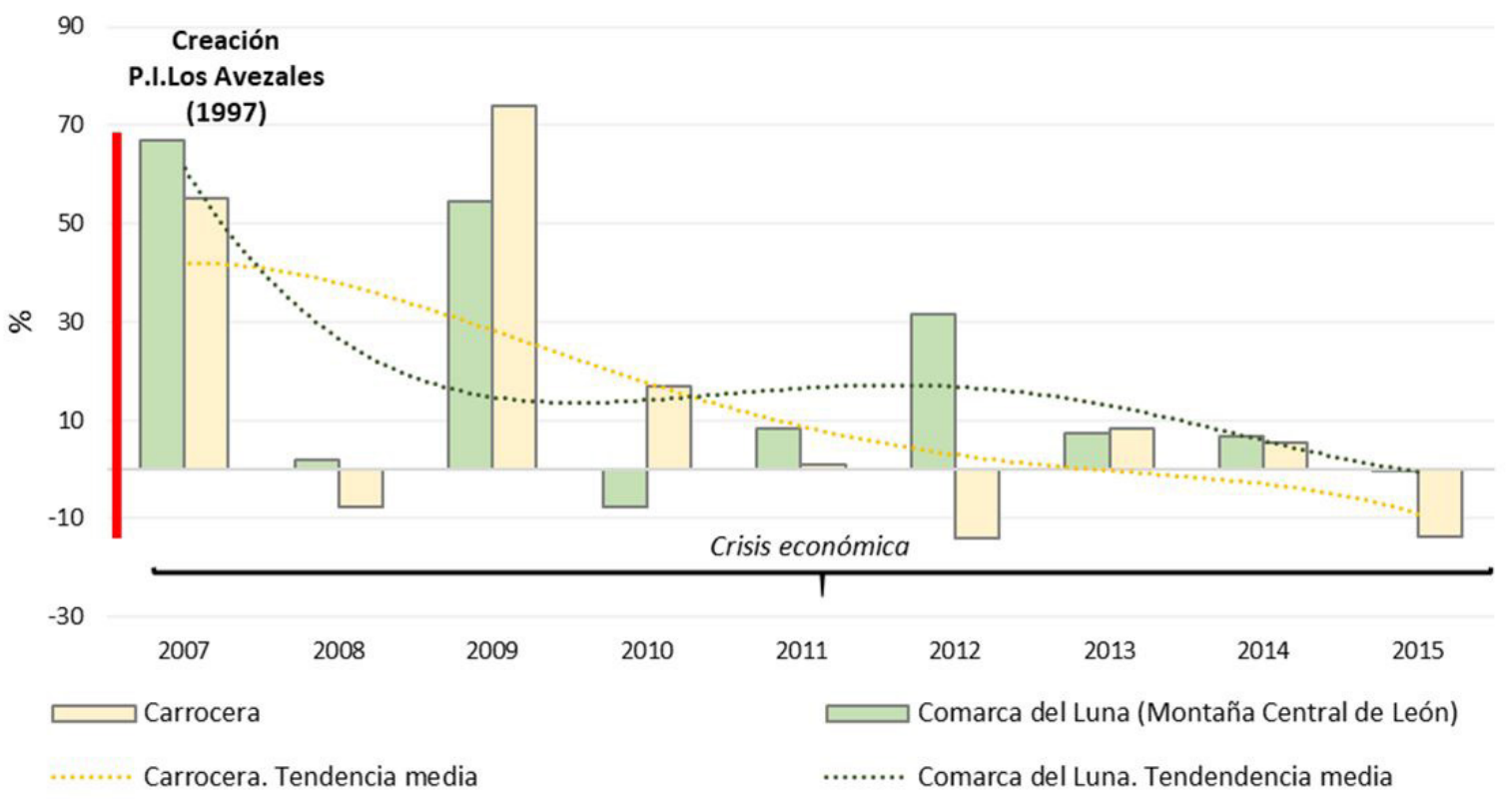

Fuente: Elaboración propia con datos de Expansión-Datosmacro (varios años).

Los ejemplos analizados ponen también de relieve que la oferta de suelo empresarial es más eficaz como elemento de oportunidad cuando se localiza en un territorio vertebrado y comunicado por una red de transportes de alta capacidad, una primera lección que se deriva de los resultados obtenidos. Tanto en los polígonos de El Bayo y Los Avezales, en El Bierzo y Montaña-Luna respectivamente, como en el caso de Villadangos, en Órbigo, la posición de estos asentamientos con respecto a autopistas y ferrocarril es decisiva para explicar, primero, la decisión política de localización de los mismos; y segundo, para reforzar las capacidades del territorio comarcal. Otra lección importante se relaciona con el papel que desempeñan en el éxito de los polígonos industriales la cercanía y buenas conexiones con núcleos urbanos y ciudades de primer orden provincial, que actúan no solo como mercados, sino también como foco difusor de sinergias expansivas, ayudando al mecanismo de la resiliencia cuando éste se activa.

Una tercera lección que se extrae de los resultados de este análisis es que la consolidación de los polígonos industriales está condicionada por los vectores que rigen la logística. Siendo desigual el grado de ocupación empresarial de unos y otros polígonos -en Villadangos se puede hablar de éxito, mientras que en El Bayo y Los Avezales la consolidación es lenta y tímida-, se confirma que la oferta de suelo urbanizado para las empresas es una ventaja que ayuda a frenar y/o a reducir el impacto de las crisis en el ámbito local; y que representa una oportunidad para superar las etapas recesivas de la economía, pues atraen inversiones cuando la tendencia es de crecimiento en entornos incluso remotos: así lo demuestran las iniciativas anunciadas en Villadangos (Centro de Proceso de Datos de Mercadona) y en El Bayo (Centro Cárnico de Alta Tecnología).

No obstante, es evidente que el suelo industrial como factor de desarrollo territorial tiene limitaciones en las áreas rurales y que se precisan otros factores que coadyuven al crecimiento económico, la creación del empleo y el freno a la despoblación. Pero también ha quedado demostrado que dónde exite este recurso las oportunidades aumentan y la capacidad de resiliencia también.

\section{Agradecimientos}

Este trabajo forma parte de los resultados del Proyecto I+D+i Retos de Investigación, Ref. RTI201895014-B-I00, Ministerio de Ciencia, Innovación y Universidades, Gobierno de España. 


\section{BIBLIOGRAFÍA Y FUENTES}

Albertos, J.M. y Sánchez, J.L. (Coords.) (2014). Geografía de la crisis económica en España.Valencia, España: Publicaciones Universitat de València.

Asociación de Geógrafos Españoles (2004). ¿Qué futuro para los espacios rurales? León, España: Universidad de León, Secretariado de publicaciones.

Benito del Pozo, P. (dir.) (2006). Suelo industrial y territorio en León. León, España: Secretariado de Publica-ciones de la Universidad de León.

Benito del Pozo, P. (2009). Crecimiento y transformación del espacio industrial en León. Boletín de la Asociación de Geógrafos Españoles, (49), 24-44.

Benito del Pozo, P. (dir.) (2011). Áreas empresariales, suelo industrial y logística: análisis y procesos en el territorio. Cizur Menor, España: AranzadiThomson Reuters.

Benito del Pozo, P., Luna, C. y Diez, F.J. (2014). Atlas de las Áreas Empresariales en España. León, España: Área de Publicaciones de la Universidad de León.

Benito del Pozo, P. (2017). Infraestructuras industriales, áreas empresariales y logística. En López, L., Escudero, R. y Placer, J. L. (coords.), Diagnóstico de la provincia de León (pp. 273-290). León, España: Área de Publicaciones de la Universidad de León.

Blasco, C. y Martínez, F.J. (2002). La planificación del suelo industrial frente al desarrollo territorial y al crecimiento económico. III Congreso Internacional de Ordenación del Territorio, Fundicot, pp.727-744.

Calderón, B. y Pascual, H. (2009). Infraestructuras de soporte en los procesos de innovación regional: los nuevos espacios productivos en Castilla y León. Boletín de la Asociación de Geógrafos Españoles, (49), 237-254.

Caravaca, I. (Coord.) (2002). Innovación y territorio. Análisis comparado de sistemas productivos locales en Andalucía. Sevilla, España: Junta de Andalucía y Universidad Pablo de Olavide.

Cortizo, J., Redondo, J.M. y Sánchez, M.J. (coord.) (2014). De la Geografía Rural al Desarrollo Local. Homenaje a Antonio Maya Frades. León, España: Área de Publicaciones de la Universidad de León.

Expansión. Datosmacro.com (varios años). https:// www.datosmacro.com/paro/espana/municipios/ castilla-leon/leon/soto-y-amio
García Pascual, F. (coord.) (2001). El mundo rural en la era de la globalización: incertidumbres y potencialidades. Madrid, España: Ministerio de Agricultura, Pesca y Alimentación y Universidad de Lleida, Serie Estudios nำ146.

González, N. y Benito del Pozo, P. (2008). Política industrial y suelo industrial: especial referencia a Castilla y León. Pecunia, (7), 101-123.

González, N. y Benito del Pozo, P. (2013-2014). Actividad empresarial y suelo industrial. Pecunia, no Extra 1, Monográfico Estudios de Economía Leonesa, pp. 17-44.

Humbert, A., Molinero, F. y Valenzuela, M. (coords.) (2011). España en la Unión Europea. Un cuarto de siglo de mutaciones territoriales. Madrid, España: Casa Velázquez.

Junta de Castilla y León (1998). La economía de Castilla y León ante el siglo XXI. Valladolid, España: Junta de Castilla y León.

Junta de Castilla y León-JCyL (2002). Plan Especial de Actuación en las Áreas Periféricas de Castilla y León, 2002-2006. Valladolid, España : Consejería de Economía y Hacienda.

López Groh, F. (dir.) (2009). La producción de suelo. De Gerencia de Urbanización a Sepes, medio siglo de historia. Madrid, España: Sepes Entidad Estatal de Suelo.

López Trigal, L., Escudero Barbero, R. y Placer Galán, J. L. (coords.) (2017). Diagnóstico de la provincia de León. León, España: Área de Publicaciones de la Universidad de León.

Manero, F. (2012). La ordenación del territorio en Castilla y León: un complejo de decisiones sujetas a desafíos permanentes. En Delgado Urrecho, J.M., (dir.). Población y poblamiento en Castilla y León (pp. 111-153). Valladolid, España: Consejo Económico y Social de Castilla y León.

Martínez, L. C. y Luengo, J. A. (2004). Concentración y difusión de la actividad industrial en Castilla y León. El papel de las infraestructuras de transporte en las dinámicas territoriales. Revista de Economía y Finanzas de Castilla y León, (9), 51-76.

Martínez, L. C. y Luengo, J.A. (2005). Localización de la actividad manufacturera en Castilla y León. Los dinamismos espaciales inducidos por la industria. Ería: Revista de Geografía, no 67, pp. 155-172. 
Maya Frades, A. (2008). El desarrollo rural como estrategia territorial: perspectiva de los espacios rurales en Castilla y León. León, España: Universidad de León, Servicio de Publicaciones.

Méndez, R. y Alonso, J.L. (eds.) (2002). Sistemas locales de empresas y redes de innovación en Castilla y León y Castilla-La Mancha. Salamanca, España: Ediciones Universidad de Salamanca.

Méndez, R. (2012). Ciudades y Metáforas: sobre el concepto de resiliencia. Ciudad y Territorio, Estudios Territoriales, (172), 215-231.

Molina de la Torre, I. (2005). La influencia de la accesibilidad en las estrategias públicas de creación de suelo industrial: el ejemplo de las campiñas meridionales de Castilla y León. En Actas XIX Congreso de Geógrafos Españoles, Santander, Universidad de Cantabria y AGE.

Moyano, E. (Coord.) (2009). Del desarrollo rural al desarrollo territorial. Reflexiones a partir de la experiencia española. Córdoba, España : Foro IESA sobre la Cohesión de los Territorios Rurales.

Pascual Ruiz-Valdepeñas, H. (2012). El significado de la industria y de las nuevas infraestructuras en la estructuración territorial. En Delgado Urrecho, J. M. (dir.). Población y poblamiento en Castilla y León. Consejo Económico y Social de Castilla y León (pp. 637-737), Valladolid, España.

Peiret, A. (1992). Localización industrial en Castilla y León. Dotación de suelo industrial. Cuadernos de Economía de Castilla y León, (2), 73-116.

Pike, A., Dawley, S. y Tomaney, J. (2010). Resilience, adaptation and adaptability. Cambridge Journal of Regions, Economy and Society, (3), 59-70.
Plan Nacional de Ortofotografía Aérea (PNOA). http:// ftp.itacyl.es/cartografia/01_Ortofotografia/ (Fecha de consulta: 21/01/2018).

Plaza Gutiérrez, J.I. (2005). Desarrollo y diversificación de las zonas rurales de España: el programa PRODER. Boletín de la Asociación de Geógrafos Españoles, (39), 399-422.

Revue Géographique de l'Est [En ligne], 57 (1-2) 2017. https://journals.openedition.org/rge/5991

Rodríguez, A. y Cortizo, J. (coords.) (2006). El Bierzo. Espacio dinámico, territorio complejo, Valladolid, España: Junta de Castilla y León.

Rodríguez Lago, J. (2012). Posibilidades de la logística en la economía leonesa. 1ㅇ Congreso Territorial del Noroeste Ibérico. Madrid: UNED (s.p.).

Sánchez, P., Gallardo, R. y Ceña, F. (2014). El medio rural andaluz frente a la crisis económica: un análisis de los factores de resiliencia territorial. Economía Agraria y Recursos Naturales, Vol. 14 (1), 27-56.

Sánchez Muñoz, M.J. y Hidalgo, C. (2017). Estrategias de desarrollo rural. En López Trigal, L., Escudero, R. y Placer, J. L. (coords.). Diagnóstico de la provincia de León (pp. 185-208). León, España: Área de Publicaciones de la Universidad de León.

Solana, M. (coord.) (2016). Espacios globales y lugares próximos. Setenta conceptos para entender la organización territorial del capitalismo global. Barcelona, España: Icaria. 